\title{
Performance Comparison of Bragg Grating-Based Optical Add-Drop Multiplexers in WDM Transmission Systems
}

\author{
Christos Riziotis and Mikhail N. Zervas* \\ Optoelectronics Research Centre, University of Southampton, \\ Southampton, SO17 1BJ, UK \\ Tel: +4423 80593141, Fax: +442380593142 \\ cr@orc.soton.ac.uk, Christos.Riziotis@osa.org \\ (*) also with Southampton Photonics Inc. \\ Chilworth Science Park, Southampton SO16 7NS,UK \\ mikhail.zervas@southamptonphotonics.com
}

Indexing Terms: Optical Add Drop Multiplexers, Bragg Gratings, Couplers, Wavelength Division Multiplexing, Communication Systems.

\begin{abstract}
The filtering performance of Bragg grating-based OADMs is studied by using theoretical system simulations. The implications of the non-optimum spectral characteristics of the coupler based OADM are quantified and compared with other filter configurations. Simulation of the performance of a non-fully optimised Add/Drop filter of this type, in an $\mathrm{IM} / \mathrm{DD}$ communication system at $40 \mathrm{~Gb} / \mathrm{s}$ transmission speed, where filter / laser source misalignments and cascading operations are present, suggests an excessive Eye-Opening Penalty of at least $0.15 \mathrm{~dB}$ compared to the case of deployment of fully optimised spectrally flat filters.
\end{abstract}




\section{Introduction}

The development of Wavelength Division Multiplexing (WDM) technology is highly critical for the successful deployment of the next generation of Broadband Optical Networks. One of the key components for WDM systems is Optical Add Drop Multiplexers (OADM). To date, Arrayed Waveguide Gratings (AWG), Thin Film Filters (TFF) and Fibre Bragg Grating (FBG) based devices form the main classes. In the last approach, narrow-band reflective Bragg gratings (for the wavelength selection), in conjunction with coupled waveguide structures (for the channel routing) have been used for the construction of four-port to provide Add and Drop functions of a particular WDM channel [1,2,3]. Spectral properties of phase Bragg gratings can be tailored to great extent [4] -in contrast with TFFs. This is primarily due to fact that TFF are transmissive devices and therefore the dispersion performance cannot be disentangled from the transmission profile. In addition due to causality, the more square the transmission profile, the larger the in-band dispersion variation [5]. FBG devices on the other hand are reflective devices and the dispersion spectrum can be defined independently from the reflection spectrum [4]. Additionally, advanced fabrication techniques have enabled the experimental demonstration of specially designed high performance gratings [6,7].

A number of different four-port Bragg grating based OADM configurations have been proposed. The simplest one, which makes direct use of the reflection properties of a fibre Bragg grating, written in a single mode fiber (Fig. 1a), is the well-known configuration with the two optical circulators [8]. This OADM implementation is frequently used as a benchmark in the sense that it uses the full potential of FBGs and provides excellent crosstalk performance and negligible back reflections. In addition, the reflection and 
dispersion characteristics of the OADM are identical to the ones of the grating. However, it should be stressed that because of the circulators this OADM suffers from relatively high insertion loss $(\sim 1 \mathrm{~dB})$, it is bulky and expensive and cannot be easily integrated.

More compact grating-based OADMs, without the use of circulators, can be classified in two main categories, namely non-interferometric and interferometric. Noninterferometric OADM architectures have been demonstrated in fibre and integrated forms. One class of this type of devices is based on grating assisted-contradirectional coupling in asymmetric couplers. In this case, a grating is used to contra-directionally couple light between two otherwise dissimilar uncoupled waveguides. In this geometry, there are in general three types of interactions involving even-even (e-e), odd-odd (o-o) eigenmode selfcoupling as well as even-odd (e-o) eigenmode resonant cross coupling [9]. In one configuration, the grating is formed in only one of the coupler's waveguides [1] (Fig. 1b) in order to minimize backreflections. This arrangement leads to many compromises and tradeoffs in order to satisfy given requirements on filter bandwidth, reflectivity and backreflections. Another non-interferometric OADM configuration (Fig. 1c) -based on a null coupler with a tilted grating in its waist- has been recently reported by Kewitsch et al. [2]. Null couplers are comprised of two dissimilar waveguides brought gradually in close proximity and finally in contact to form the waist. The devices are actually based on adiabatic mode transformation taking place along the coupler arms and their waist. A short period UV-written tilted grating can provide resonant contra-directional coupling and add/drop function. We have shown recently $[9,10]$ that with appropriate design this device can exhibit superior fully optimised performance and with spectral characteristics identical to them of the deployed grating.

In the other category, interferometric OADMs rely on the interference between two optical signals reflected by Bragg gratings that are incorporated into various interferometric 
optical arrangements. It is well known that a configuration based on a perfectly matched Mach-Zehnder Interferometer (MZD) (Fig 1d), with identical gratings in each arm, can potentially result in an ideal performance [11]. However, grating mismatches and interferometer-arm imperfections compromise the OADM performance severely, resulting in strong backreflections and spectral distortions. In his case, careful post-processing and trimming is required [12]. A recently proposed alternative configuration is based on symmetric, full (100\%) couplers (SFC) (Fig. 1e) with the Bragg grating placed in the coupler waist to reflect the two lowest-order eigenmodes [3]. In the MZI OADM any imbalances of the order of one wavelength can severely degrade its performance because the interference happens between different waveguides. But in the SFC configuration its operation is based on the beating between the normal modes of the coupled waveguide structure. Thus, the allowed imbalances are now of the order of the coupling length, making this configuration more robust. The well-developed fused-fibre coupler technology together with advanced UV writing techniques for the inscription of fibre Bragg gratings could enable the fabrication of this type of device. However the grating has to be placed precisely in the correct position for optimum operation. This position is asymmetric along the coupler waist making thus the device asymmetric in operation.

This paper analyses the implications of the inherently non-optimum spectral characteristics of this device [13]. The spectral response of the OAM is modeled for different configurations depending on the grating positioning in the coupler waist. Using system simulations, the degradation of $40 \mathrm{~Gb} / \mathrm{s} \mathrm{NRZ} \mathrm{modulated} \mathrm{signal} \mathrm{is} \mathrm{estimated} \mathrm{when} \mathrm{these}$ OADMs are deployed. We quantify the distortion effects by estimating the eye-opening penalty (EOP) after passing the signal through cascaded Add and Drop actions. The effect of filter and laser source misalignments is also considered for the estimation of the excess system EOP. 


\section{Full-Coupler Based OADM Principle of Operation}

The device is shown schematically in Figure 2, together with the power evolution along the full coupler (Fig 2a). The total length of the coupler is $L_{c}=Z_{c} / 2$ where $Z_{c}$ is the beat length between the even and odd supermodes of the coupler. If $n_{e}$ and $n_{0}$ are the effective refractive indices of the even and odd supermodes respectively, then the beatlength is defined as $Z_{c}=\lambda /\left(n_{e}-n_{0}\right)$, where $\lambda$ is the operating wavelength.

Figure 2 demonstrates that a stream of WDM channels enters through Port 1 and the channel at wavelength $\lambda_{1}{ }^{\text {DROP }}$ is reflected by the grating and dropped at Port 2 . The grating is placed asymmetrically within the waist so that its effective reflecting point at the wavelength

of interest $\lambda_{1}{ }^{\text {DROP }}$ coincides with the half point $(\mathrm{M})$ of the coupler. At this point the two supermodes of the coupler are reflected and their destructive interference at Port 1 guarantees that all the power at the wavelength of interest will appear at the Drop Port 2. It should be stressed that the two modes have different propagation constants and their corresponding reflection spectra are spectrally displaced with respect to each other and, therefore partially overlapping. The bandwidth of the Drop channel is determined by this overlap.

The analysis and modelling of this interferometric device can easily be performed by decomposing the propagating fields into the even and odd supermodes of the coupler and by calculating the interference between them. Symmetry consideration guarantees that these modes do not cross couple energy by scattering from the grating (assuming always that the grating is written perpendicular and placed symmetrically with respect to the waist axis). However for the analysis here we applied a more rigorous method, which follows closely the 
one presented in [9]. As demonstrated in Figure 2, in order to configure the OADM optimally the grating has to be placed accordingly by taking into account its penetration depth -at the central wavelength of the dropped channel.

Next, we briefly introduce the time delay and equivalent penetration depth of the reflected light. If $\theta=$ phase $(r)$ is the phase of the reflection coefficient $r$ of the grating then the time delay for reflected light is:

$$
\tau=\frac{d \theta}{d \omega}=\frac{d \theta}{d k} \cdot \frac{1}{c_{o}}
$$

where $k=\frac{\omega}{c_{o}}=\frac{2 \pi}{\lambda}$ and $c_{o}$ is the speed of light in vacuum. The equivalent penetration depth of a particular wavelength can be estimated by considering the length, which corresponds to half the time delay. Thus

$$
L_{p}=\frac{1}{2} c \cdot \tau=\frac{1}{2} \cdot \frac{c_{o}}{n} \cdot \frac{d \theta}{d k} \cdot \frac{1}{c_{o}}=\frac{1}{2 \cdot n} \cdot \frac{d \theta}{d k}
$$

where $c$ is the speed of light in the waveguide-grating and $n$ is the average refractive index of the waveguide.

For a typical example of the coupler based OADM we estimate its full spectral response. Figure $3 a$ shows the reflectivity spectra of the even and odd modes $R_{e}$ (dashed line) and $R_{0}$ (dashed-dotted line) centered respectively at wavelengths $\lambda_{e}, \lambda_{0}$, and also the Drop reflectivity spectrum $\mathrm{R}_{\mathrm{DROP}}$ (thick solid line). The relative spectral displacement is:

$$
\Delta \lambda=\left|\lambda_{e}-\lambda_{o}\right|=2 \cdot\left(n_{e}-n_{o}\right) \cdot \Lambda_{o}=\frac{2 \cdot \lambda \cdot \Lambda_{o}}{Z_{c}}
$$

where $\Lambda_{o}$ is the period of the grating. Increasing $Z_{c}$ can minimize the spectral displacement and improve the $\mathrm{R}_{e}, \mathrm{R}_{\mathrm{o}}$ overlap. Figure $3 \mathrm{~b}$ shows the group time delay of the reflection spectrum for the even and odd normal modes. They are also spectrally displaced by $\Delta \lambda$. The 
solid line represents the average time delay of both modes. The equivalent penetration depth, which is related to group time delay by Equation (2), varies considerably with wavelength. The time delay in transmission of symmetric gratings is known to be identical to the one in reflection. Time delay variations in transmission, however, correspond effectively to varying coupler length. These dispersive effects are expected to affect considerably the spectral response in all ports of the OADM.

Figure 4 shows a typical full spectral response in all ports of a SFC OADM. The grating is arranged so that its effective reflection point around the Bragg wavelength coincides with $\mathrm{M}$ and therefore drops efficiently around this wavelength. Away from the Bragg wavelength, however, the time delay increases considerably (Fig. 3b) and the effective reflection point departs considerably from $M$. As a result, the reflected light appears partially back in Port 1 (Fig. 4a). This backreflection becomes more severe in the case of a stronger grating.

The spectra in Figure $4 \mathrm{~b}$ show the transmission response in Port 4 and Port 3 . Under ideal operation conditions, no light should appear in Port 3. However, the power leaking into this port is due to the additional grating dispersion in transmission, which results in variation of the effective coupler length. As expected, the spectral response of the leaking Port 3 follows closely the average time delay variation in Figure $3 b$.

It is obvious that when the device is optimised for channel dropping at Port 2, the channel adding at Port 3 is severely degraded. For simultaneous Add-and-Drop function the device can be configured in a symmetrical manner by placing the Bragg grating at the center of the coupler waist $\left(\mathrm{L}_{1}=\mathrm{L}_{3}\right)$. This way the device will exhibit identical but compromised Add and Drop actions (Fig. 2c) 


\section{Modelling of Spectral Responses of the Full Coupler Based OADM}

In this section we calculate the spectral response of a typical coupler based OADM device, for different configurations and operation modes. A full coupler with length $20 \mathrm{~mm}$ and a uniform waist length $\mathrm{L}_{\text {waist }}=\mathrm{L}_{1}+\mathrm{L}_{2}+\mathrm{L}_{3}=10 \mathrm{~mm}$ is considered. The deployed grating has a typical reflectivity $35 \mathrm{~dB}$ with length $\mathrm{L}_{\mathrm{g}}=5.2 \mathrm{~mm}$, amplitude of refractive index modulation $\Delta \mathrm{n}=3.65 \times 10^{-4}$, and sine-apodisation profile. By modelling first the spectral response of the grating we estimate the penetration depth at the operating wavelength $\lambda_{1}=1.55 \mu \mathrm{m}$ to be $\mathrm{Lp} \sim 0.965 \mathrm{~mm}$

It is important to stress that despite the fact that the grating response is symmetric, the coupler based OADM response is different under Add/Drop action depending on the relative grating position. There are two main OADM configurations that we study. The first one (shown in Figure 2b) is optimised for Drop action -Optimised (O)- by placing the grating inside the waist region asymmetrically with respect to the mid-point $M$ so that $\mathrm{L}_{\mathrm{l}}+\mathrm{L}_{\mathrm{p}}=\mathrm{L}_{\text {wais }} / 2$. It is obvious that in this case the Add action is quite degraded -Degraded (D)- due the fact that the effective reflection point on this grating side is well off the waist mid-point $M$. In the second configuration (shown in Fig. 2c), the grating is placed symmetrically around the waist mid-point $M$ and the both effective reflection points are on either side of $M$. Due to symmetry this configuration provides identical although compromised -Compromised (C)-Add \& Drop operation.

Figure 5 shows the reflectivity spectra in linear scale for all those different responses. Together is plotted the reflection spectrum of the deployed Bragg grating. The narrowing of the OADM reflection is obvious as explained before. Figure 6a gives the intra-band group time delay of all those responses and Figure $6 \mathrm{~b}$ gives the corresponding intra-band 
dispersion, calculated as $D=\frac{d \tau}{d \lambda}$. From Figure 6 it is realized that the time delay and dispersion are almost identical for all different modes of operation within the bulk of the reflection band. The main difference occurs right at the edges of the reflection bands. Figure 5 , however, shows that the reflectivity spectra are distinctively different for the considered modes of operation. Any differences in the system response of these devices are expected to be primarily due to the reflectivity spectra.

Based on these modeled spectral responses of the OADM filter we estimate its performance in a communication system, by examining the pulse distortion effects and quantifying them by measuring the closure of the resulted eye diagram. The transfer function of the filters is formed by both the amplitude and phase response. Deployment of degraded and compromised OADMs induces excess loss to the system leading to excess system penalty. We concentrate our study here only on the distortion effects due to filter's spectral properties and we neglect the additional loss, which can always be compensated by use of optical amplifiers [14].

In Figure 7 we introduce some representative Add/Drop operations for the characterization of the OADM performance. Figure 7a describes a single network node where a WDM channel can either be Added or Dropped, or can experience a cascaded Dropand-Add action with the use of a switch. In the node, the Dropped channel can be transmitted to the node's final user (cross state of switch) or through a bypass operation (bar state of switch) can be added again to the optical stream leading thus to a two-stage cascade in a single OADM. In a real access network a sequence of optical nodes can lead to a number of cascaded Drop-and-Add operations [15]. Figure $7 \mathrm{~b}$ describes the Add-and-Drop cascaded operation for an OADM, which can occur in different nodes of a WDM network. The combined action of two OADMs gives the Add-and-Drop function where a particular WDM 
channel is added in a node and then dropped in another node in the network. If we consider that these two OADMs are SFC type devices, then depending on their individual configurations and also the relative orientation of their Ports we can get a set of different combinations and different overall spectral responses for the two stage cascaded $A d d /$ Drop action. Namely, 'Optimised Drop \& Degraded Add' (O-D), 'Compromised Drop and Add' (C-C), 'Degraded Drop \& Degraded Add' (D-D) are some interesting combinations.

Figure 8 shows some of the representative transfer functions of the filtering operations described in Figure 7. The transfer functions, which correspond to the reflectivity spectra in Figure 5, are plotted in Figure 8 with the same line code for comparison. The magnitude of the transfer function is the square root of reflectivity. Together are plotted also the $(C-C),(O-D)$ and $(D-D)$ transfer functions. We can notice that despite their different average level, which should lead to different overall insertion loss, the shapes of the (O-D) and $(C-C)$ responses are the same. Consequently it is expected that they suffer form identical intra-band distortion effects. This will be confirmed in the next section using system simulations, which take also the phase response of the filters into account.

\section{Characterization of OADMs in a Communication System}

The quality of the spectral characteristics of an OADM is very critical in high-speed WDM systems where the dense packing of channels requires the bandwidth (BW) of these selective filters to be the minimum possible in order to accommodate the bandwidth of a signal with given Bit Rate. With such a high filling factor, defined as the ratio of signal bandwidth and filter bandwidth, distortion effects can arise from any spectral-shape degradation of the filters. The implications of the non-optimum intra-band spectral characteristics of the 
interferometric OADM are examined here by estimating the EOP in an Intensity Modulation / Direct Detection (IM/DD) Communication System (Fig. 9). The simulated system is based on the SONET standards and the employed electrical filters at the transmitter and receiver are 4-order Bessel filters with 3-dB bandwidth $75 \%$ of the Bit Rate. A chirp-less input is assumed into the OADMs by employing a balanced Mach-Zehnder modulator. The $3 \mathrm{~dB}$ bandwidth of the WDM filters we considered and simulated in the previous section is $\sim 0.7 \mathrm{~nm}$ and the distortion effects are examined here for a non-return to zero (NRZ) modulated signal with and $40 \mathrm{~Gb} / \mathrm{s}$ transmission speed, which accordingly occupies almost all this bandwidth. The signal is a pseudo random bit sequence (PRBS) of $2^{7}$ bits. Figure 10 shows the reflection spectra -in logarithmic scale- of the deployed Bragg grating together with the three different OADM responses. The spectrum of the modulated signal is also plotted for comparison. We examine initially the distortion effects for a single and double cascaded operations (Fig. 7), as a function of misalignment between the center frequency of the effective filter and the center frequency of the modulated signal spectrum. The results obtained here could be transferred to any bit rate and equivalent employed filters, with the same filling factor.

Figure 11 gives the EOP for a single Add or Drop action. As expected the deployed Bragg grating is superior, given also its broader bandwidth. The optimised (O) Drop is slightly degraded compared to the BG case. We can notice also from the graph that for the misalignment range of $[-0.2 \mathrm{~nm}$ to $+0.2 \mathrm{~nm}]$ the non-optimum OADM responses leads to an EOP higher by at least $0.15 \mathrm{~dB}$.

Figure 12 shows the EOP for the Add-and-Drop operation described in Figure 7b. The 'Optimised Drop \& Degraded Add' $(O-D)$ and the 'Compromised Drop and Add' (C-C) configurations exhibit exactly the same EOP relation versus the filter misalignment, as predicted from their spectral shape. So we can conclude that the overall Add-and-Drop 
operation of a particular SFC OADM is independent of the individual responses and consequently insensitive to the grating arrangement in the coupler waist. This is a direct consequence of the linearity and reciprocity of the composite OADM four port device [16].

In a large-scale network with a number of different nodes, the worst-case scenario combination of Degraded Drop-Degraded Add (D-D) operation can occur which as shown in Figure 12, suffers considerably higher EOP. By comparing Figures 11 and 12 it is clear that even a two stage cascading makes the difference between optimum and degraded filters much more prominent especially for misalignments above $\pm 0.05 \mathrm{~nm}$. The use of degraded filters in this two-cascade operation results in a reduction of the allowed range of filter-laser misalignments by a factor of two, in order to keep the EOP below $1 \mathrm{~dB}$.

As discussed in the introduction, OADMs based on non-interferometric configurations like the optical circulator type (Fig. 1a), or the null coupler with a tilted grating (Fig. 1c) exhibit intra-band spectral characteristics identical of them of the deployed Bragg grating. Consequently deployment of this type of filters in a WDM network should be associated with the performance curves (in Figures $11 \& 12$ ), which represents the use of the single Bragg-grating (BG).

Finally to determine the tolerance of the different OADM filters in cascaded operation we estimated the EOP for all the considered different responses of the SFC OADM device as a function of the cascade count. The filters are considered perfectly aligned with each other and aligned also with the modulated signal spectrum. Two different studies were performed. First we considered the actual transfer function of each filter, which contains both the amplitude and phase response. In the second case we neglected the phase response of the filters, concentrating thus only on the distortion effects due to reflectivity spectral shape. The results for these two cases are concluded in Figures 13 and 14 respectively. By comparing Figures 13 and 14 we conclude that the number of cascades in order to keep the 
EOP bellow $1 \mathrm{~dB}$ is limited mainly by dispersion. When only the reflectivity is considered (Fig. 14) we can notice that for the Optimised (O) or the Bragg Grating (BG) cases the EOP is kept well below $0.2 \mathrm{~dB}$ even after 32 cascades, while in contrast, is increased exponentially for the non-optimum filter responses. Deployment of specially designed, dispersionless Bragg gratings [7] could greatly improve the cascadability of those OADMs. The advantage however would be much greater for spectrally optimised OADMs as demonstrated in Figure 14.

\section{Conclusions}

By performing system simulations we have studied the Intra-Band characteristics of the main representatives of Bragg-grating based OADM devices, in the frame of high bit rate applications in DWDM networks. Is shown that even small spectral degradation in the OADMs amplitude response degrades their filtering performance quite drastically, especially when filter-laser misalignments and cascaded operations are present in to the system. A typical degraded response of the full coupler based OADM leads to an excessive EOP of at least $0.15 \mathrm{~dB}$ compared with the case of spectrally flat deployed filters. Depending on the scale of the application system these device limitations could be critical for the overall system performance and together with the additional insertion losses associated with a nonoptimum OADM response, should be taken into account in the design process.

\section{Acknowledgment}

This work was supported in part by the UK EPSRC project PHOTON. 


\section{References}

[1] DONG, L., HUA, P., BIRKS, T.A, REEKIE, L., RUSSELL, P.ST.: 'Novel add-drop filters for wavelength-division multiplexing optical fiber systems using a Bragg grating assisted mismatched coupler', IEEE Photon. Technol. Lett., 1996, 8(12), pp. 1656-1658

[2] KEWITSCH, A.S., RAKULJIC, G.A., WILLEMS, P.A., YARIV, A.: 'All fiber zero insertion loss add drop filter for WDM All fiber zero insertion loss add drop filter for WDM', Opt. Lett., 1998, 23(2), pp.106-108

[3] BAKHTI, K., SANSONETTI, P., SINET, C., GASCA, L., MARTINEAU, L., LACROIX, S., DAXHELET, X., GONTHIER, F.: 'Optical add-drop multiplexer based on UV written Bragg gratings in a fused 100\% coupler', Electron. Lett., 1997, 33(9), pp. 803804

[4] FECED, R., ZERVAS, M.N., MURIEL, M.A.: 'An efficient inverse scattering algorithm for the design of nonuniform fiber Bragg gratings', IEEE J. Quantum Electron., 1999, 35(8), pp. $1105-1115$

[5] LENZ, G., EGGLETON, B.J., GILES, C.R., MADSEN, C.K., SLUSHER, R.E.: 'Dispersive properties of optical filters for WDM systems', IEEE J. Quantum Electron., 1998, 34(8), pp. 1390-1402

[6] LOH, W.H., COLE, M.J., ZERVAS, M.N., BARCELOS, S., LAMING, R.I.: 'Complex grating structures with uniform phase masks based on the moving fiber-scanning technique' Opt. Lett., 1995, 20(20), pp. 2051-2053 
[7] IBSEN, M., PETROPOULOS, P., ZERVAS, M.N., FECED, R.: 'Dispersion-free fiber Bragg gratings', Optical Fiber Communication Conference, OFC'2001, March 2001, Anaheim, paper MC1

[8] JONES, K.P., CHADRY, M.S., SIMEONIDOU, D., TAYLOR, N.H., MORKEL, P.R.: 'Optical wavelength add-drop multiplexer in installed submarine WDM network', Electron. Lett., 1995, 31(24), pp. 2117-2118

[9] RIZIOTIS, C., ZERVAS, M.N.: 'Design considerations in Optical Add/Drop Multiplexers based on grating-assisted null couplers', Journal of Lightwave Technology, 2001, 19(1) pp.92-104

[10] RIZIOTIS, C., ZERVAS, M.N.: 'Optimisation of OADMs based on grating assisted mode conversion in null couplers', $25^{\text {th }}$ European Conference on Optical Communication, ECOC'99, September 1999, Nice, France, paper TuD1.6.

[11] JOHNSON, D.C., HILL, K.O., BILODEAU, F., FAUCHER, S.: 'New design concept for a narrowband wavelength-selective optical tap and combiner', Electron. Lett., 1987, 23(13), pp. 668-669

[12] KASHYAP, R., MAXWELL, G.D., AINSLIE, B.J.: 'Laser-Trimmed four-port bandpass filter fabricated in single-mode photosensitive Ge-doped planar waveguide', IEEE Photon. Technol. Lett., 1993, 5(2), pp. 191-194 
[13] RIZIOTIS, C., ZERVAS, M.N.: 'Characterization of Optical Add Drop Multiplexers in high bit rate WDM networks', 2nd International Symposium on Communication Systems, Networks and Digital Signal Processing (CSNDSP), July 2000, Bournemouth, UK, pp. 102107

[14] KHRAIS, N.N., ELREFAIE, A.F., WAGNER. R.E., AHMED S.: 'Performance degradation of multiwavelength optical networks due to laser and (de)multiplexer missalignement', IEEE Photon. Technol. Lett., 1995, 7(1), pp. 1348-1350

[15] SALEH, A.A.M., SIMMONS, J.M.: 'Architectural principles of optical regional and metropolitan access networks', Journal of Lighwave Technology, 1999, 17(12), pp. 24312448

[16] HAUSS, H.A.: 'Waves and Fields in Optoelectronics'. Englewood Cliffs, PrenticeHall, 1984 


\section{FIGURE CAPTIONS}

Figure 1. Schematic of the main OADM configurations based on Bragg gratings in coupled waveguide structures.

Figure 2. Schematic of the full coupler interferometric OADM. a) Power evolution along the full coupler b) Asymmetric configuration for optimised Drop action, c) Symmetric grating arrangement with compromised OADM performance

Figure 3. a) Reflectivity spectrum for Drop action in a full coupler OADM. The Drop action is formed by the partial overlap of the reflection responses of the two normal modes. b) Overlap of the group time delay responses of the even and odd modes. The solid line represents the average group delay of both responses.

Figure 4. Typical full spectral response of the SFC OADM. a)Reflection spectrum, b)Transmission spectrum

Figure 5. Comparison of the typical reflectivity spectra, in linear scale, for the deployed Bragg grating ( $B G)$, Optimised (O), Compromised (C) and Degraded (D) actions.

Figure 6. a) Comparison of the intra-band typical group time delay characteristics for the $(B G),(O),(C)$ and $(D)$ responses. The time delays for all the cases were normalized to zero value at the center of the filter. b) Comparison of the intra-band dispersion characteristics for the same responses. 
Figure 7. a) Schematic of a single node where a channel is added to the input optical stream or dropped from it through an OADM. b) Schematic of a fundamental cascaded Add-andDrop action in an optical network.

Figure 8. Comparisons of magnitude of the reflection coefficient for single pass and cascaded Add-and-Drop actions.

Figure 9. Block diagram of the simulated test-bed for the characterization of OADMs.

Figure 10. Filling of the bandwidth of the different filter responses with the spectrum of NRZ $40 \mathrm{~Gb} / \mathrm{s}$ PRBS signal.

Figure 11. EOP as a function of filter / laser source misalignment for a single Drop or Add action.

Figure 12. EOP as a function of filter / laser source misalignment for a cascaded Add-andDrop action, for representative filters combinations.

Figure 13. Effect of OADM cascade on EOP performance.

Figure 14. Effect of $O A D M$ cascade on EOP performance when only the reflectivity response of the filters is considered. 
(a)

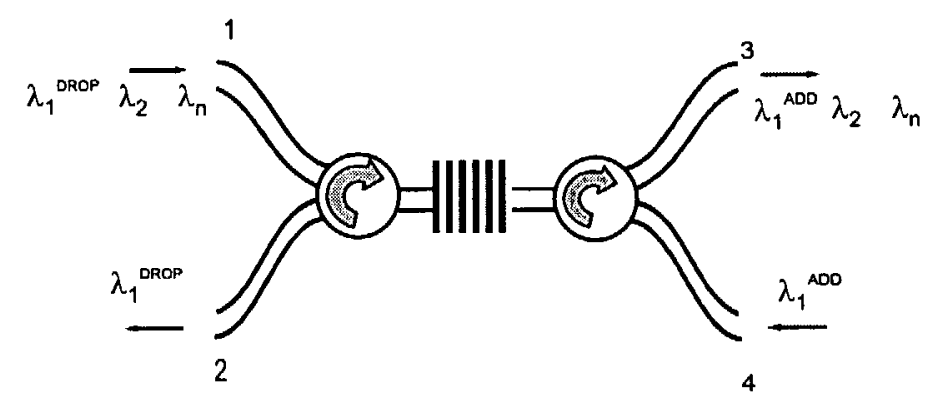

(b)

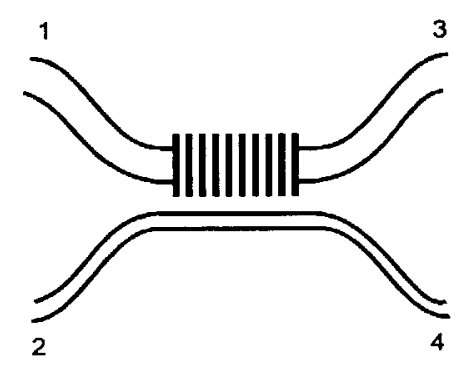

(c)

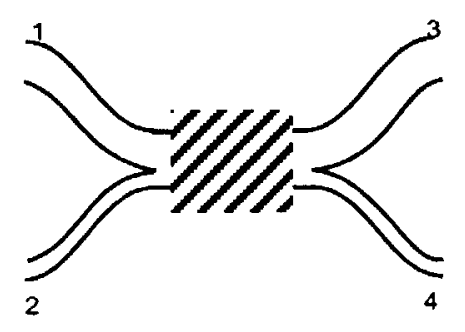

(d)

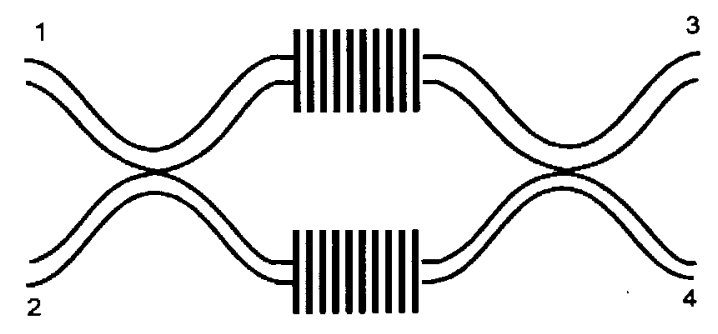

(e)

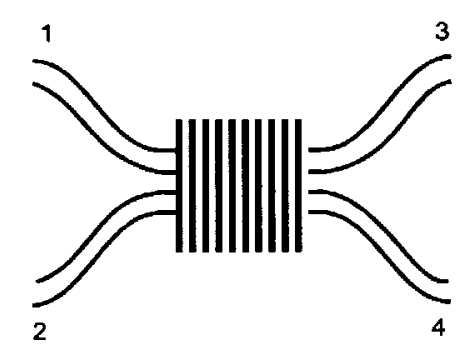

Fig. 1 
(a)

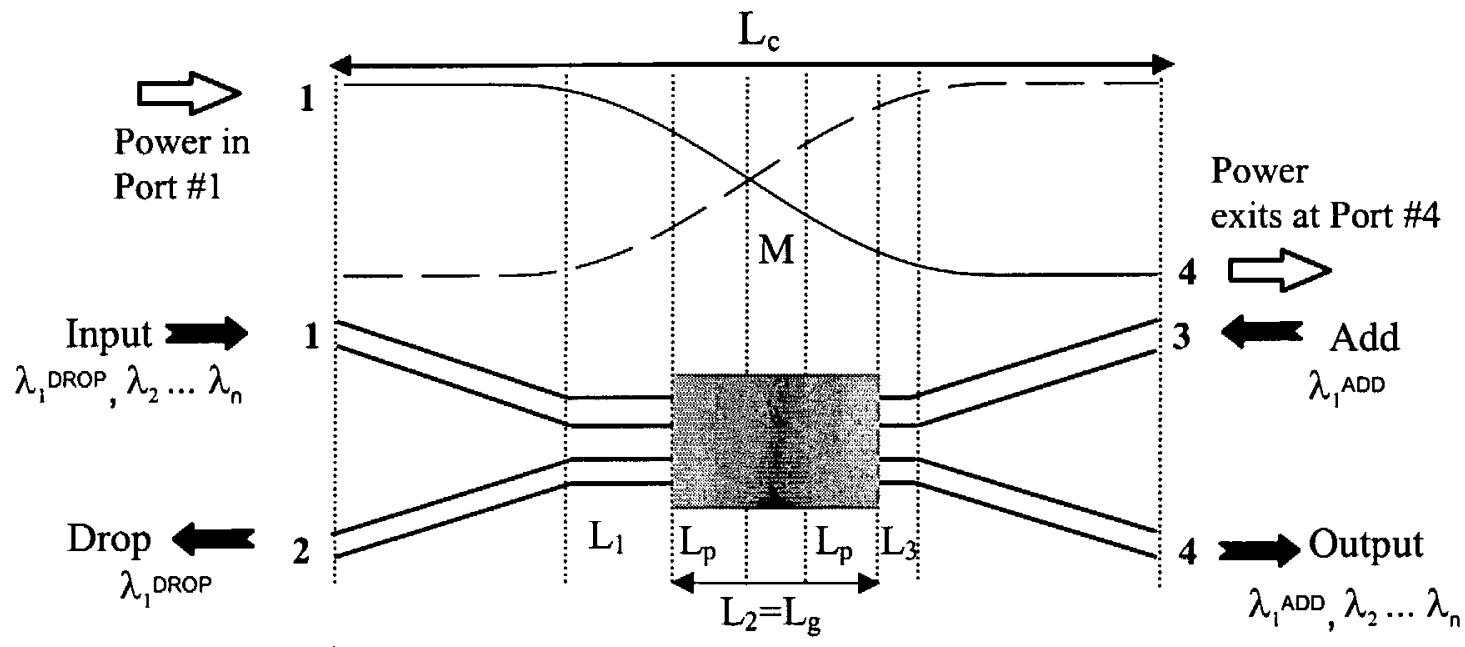

(b)

(c)

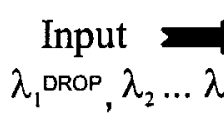

Drop

$\lambda_{1}$ DROP

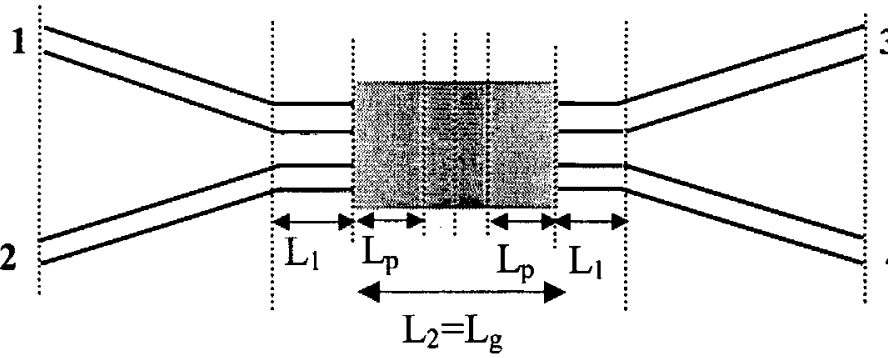

$4 \Rightarrow$ Output

$\lambda_{1}{ }^{\mathrm{ADD}}, \lambda_{2} \ldots \lambda_{\mathrm{n}}$

Fig. 2 
(a)

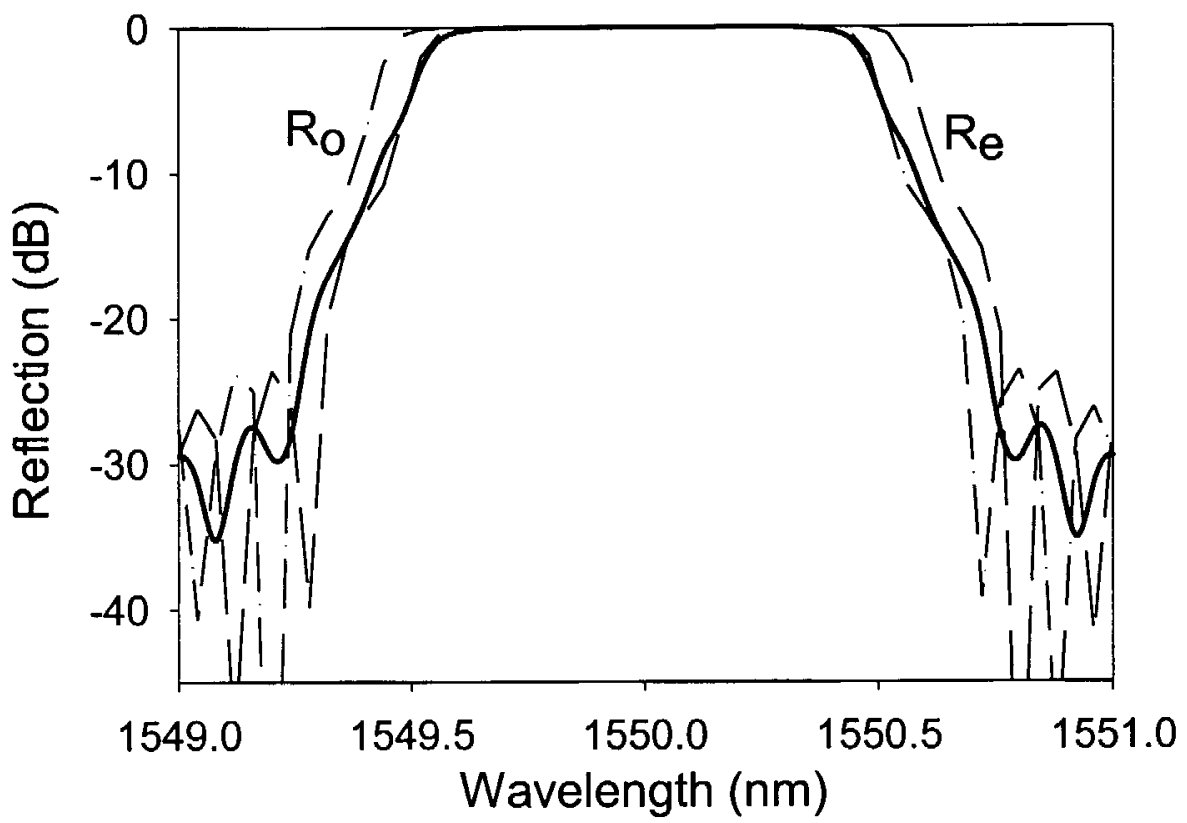

(b)

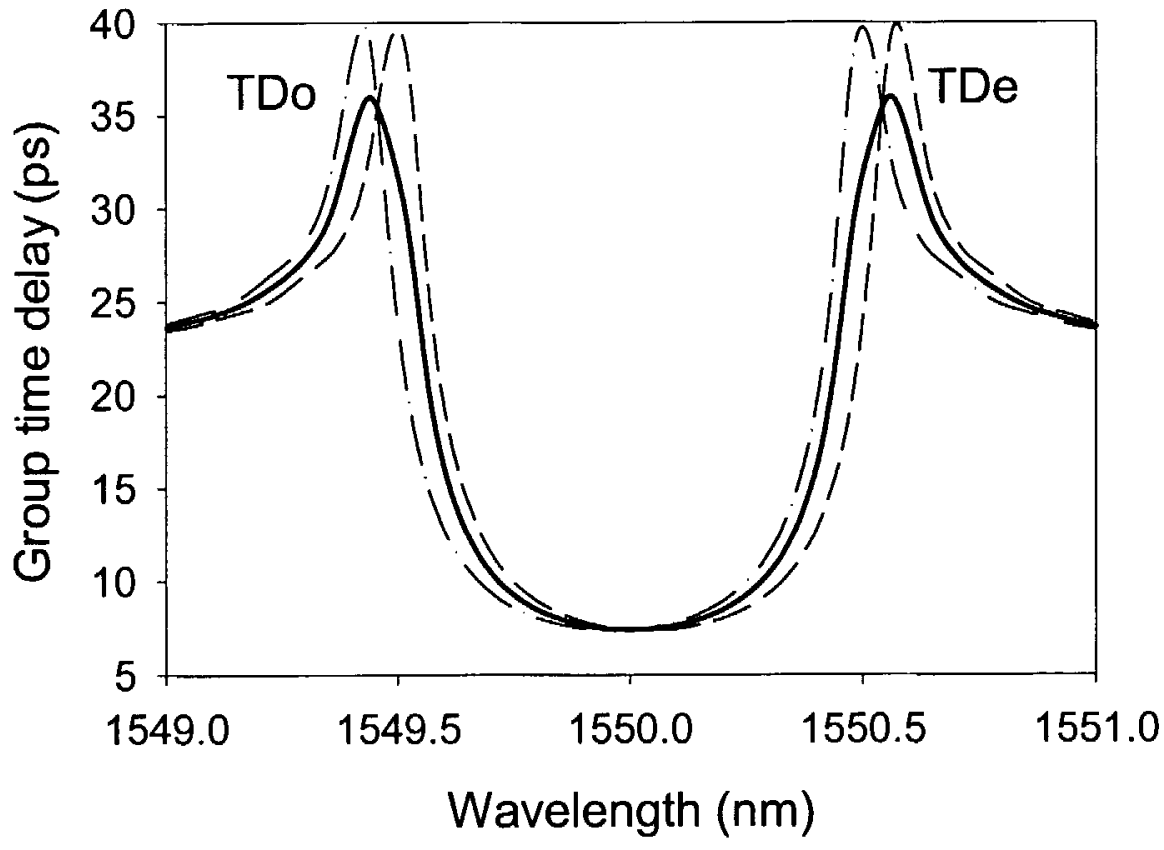

Fig. 3 
(a)

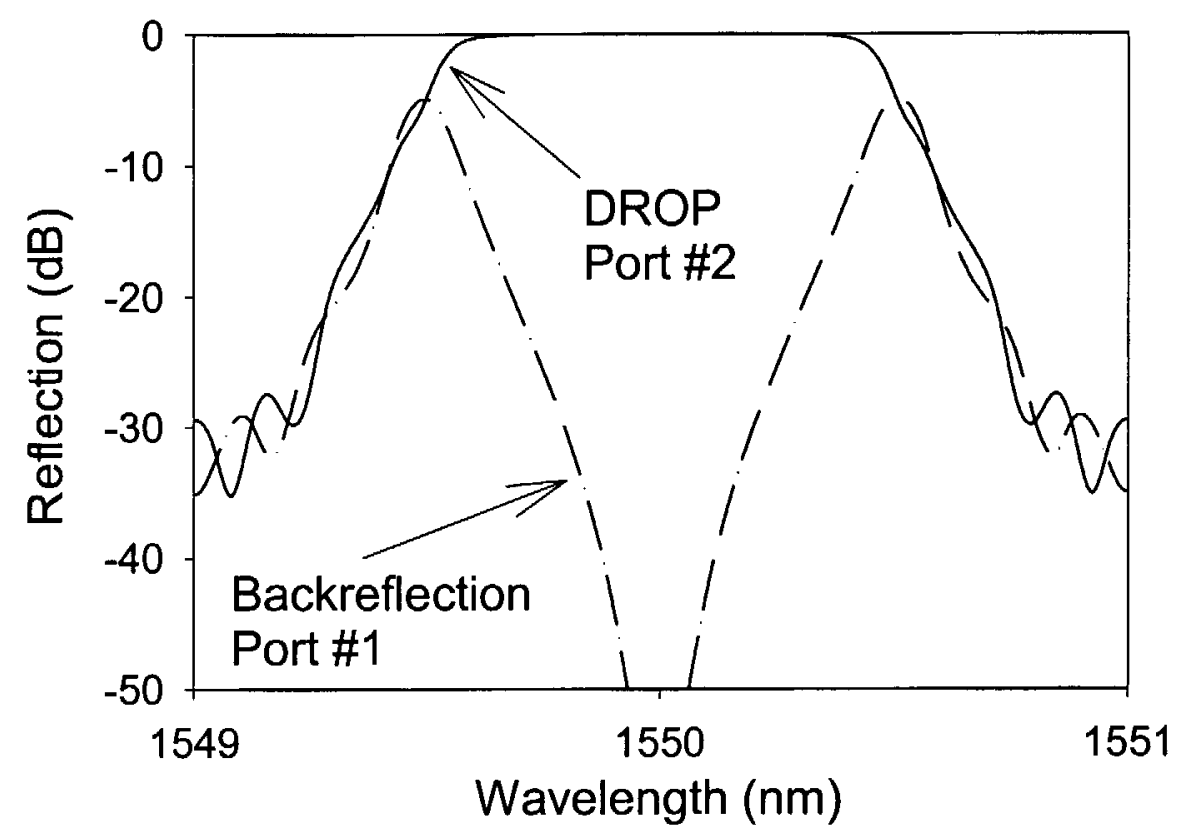

(b)

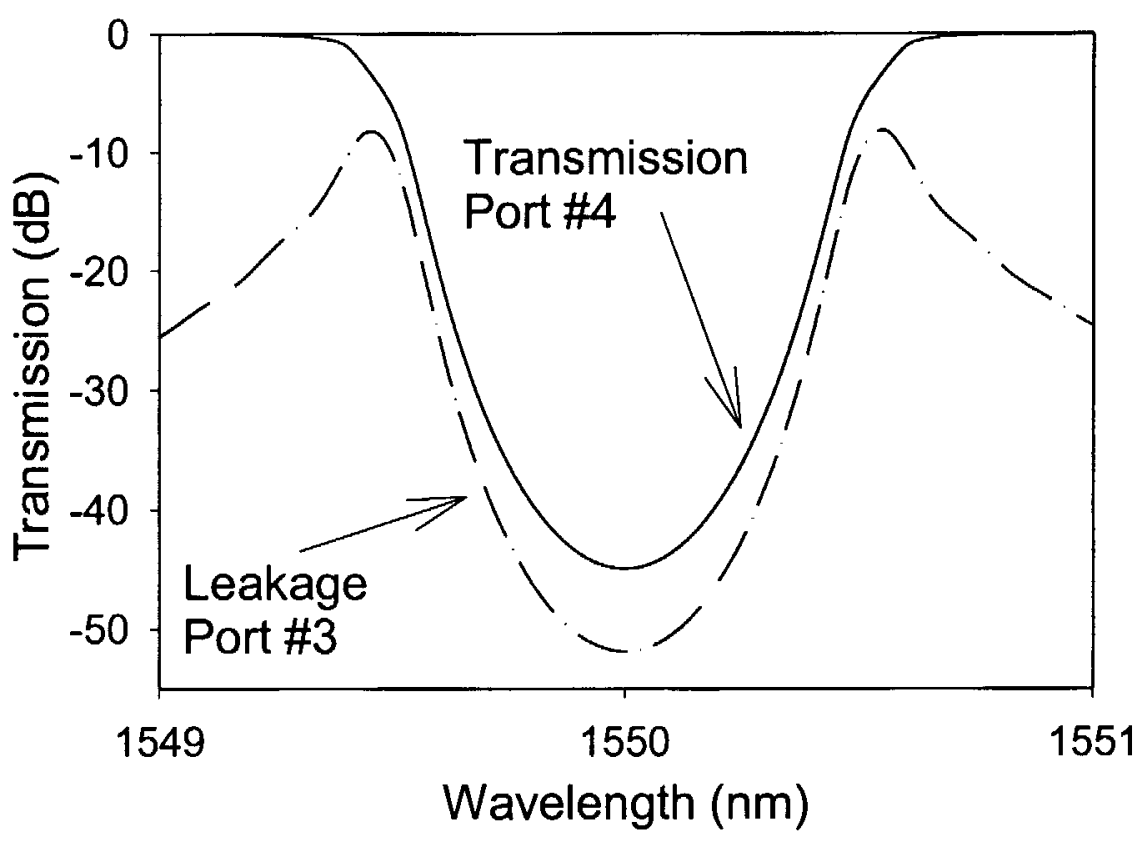

Fig. 4 


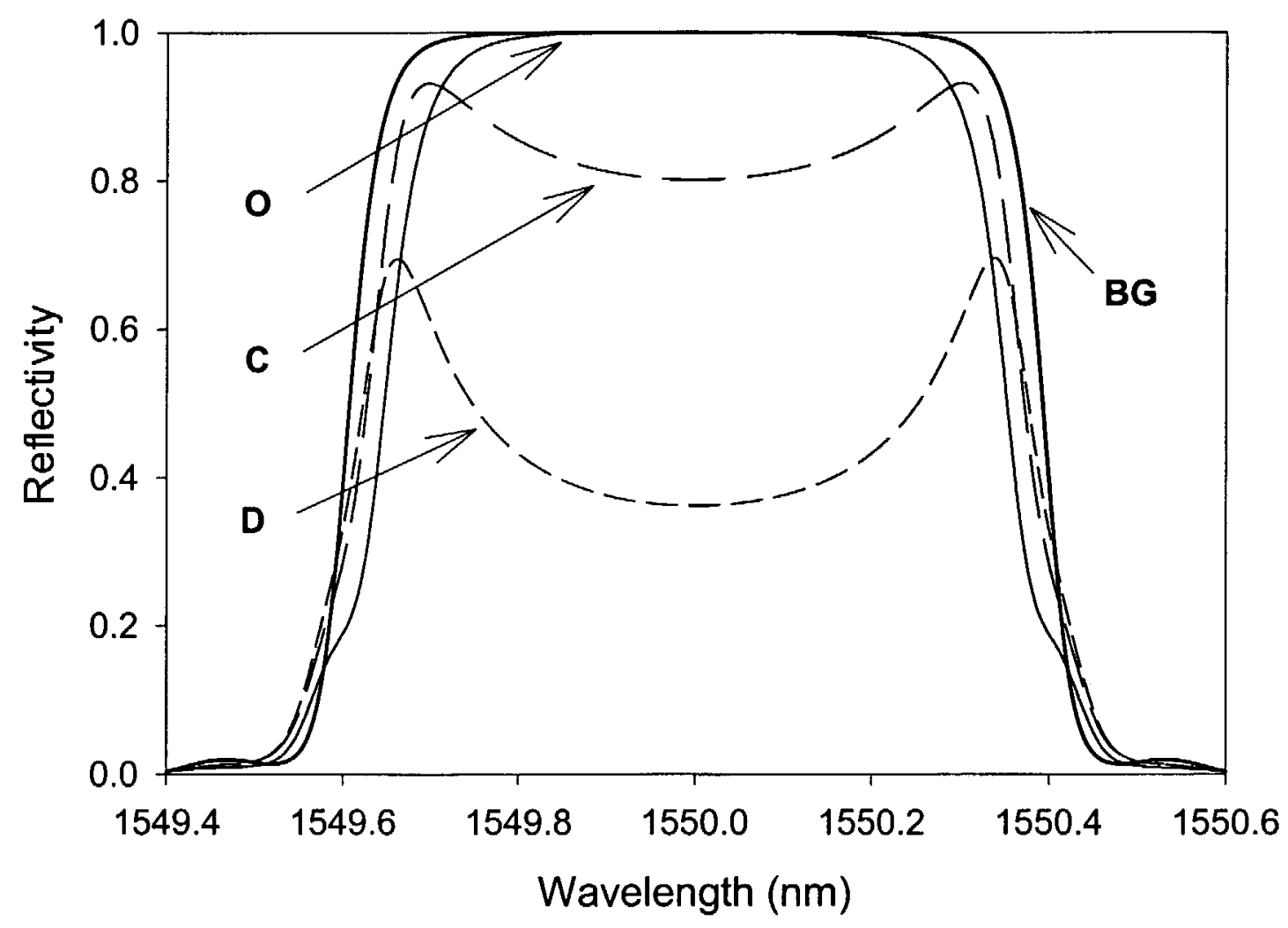

Fig. 5 


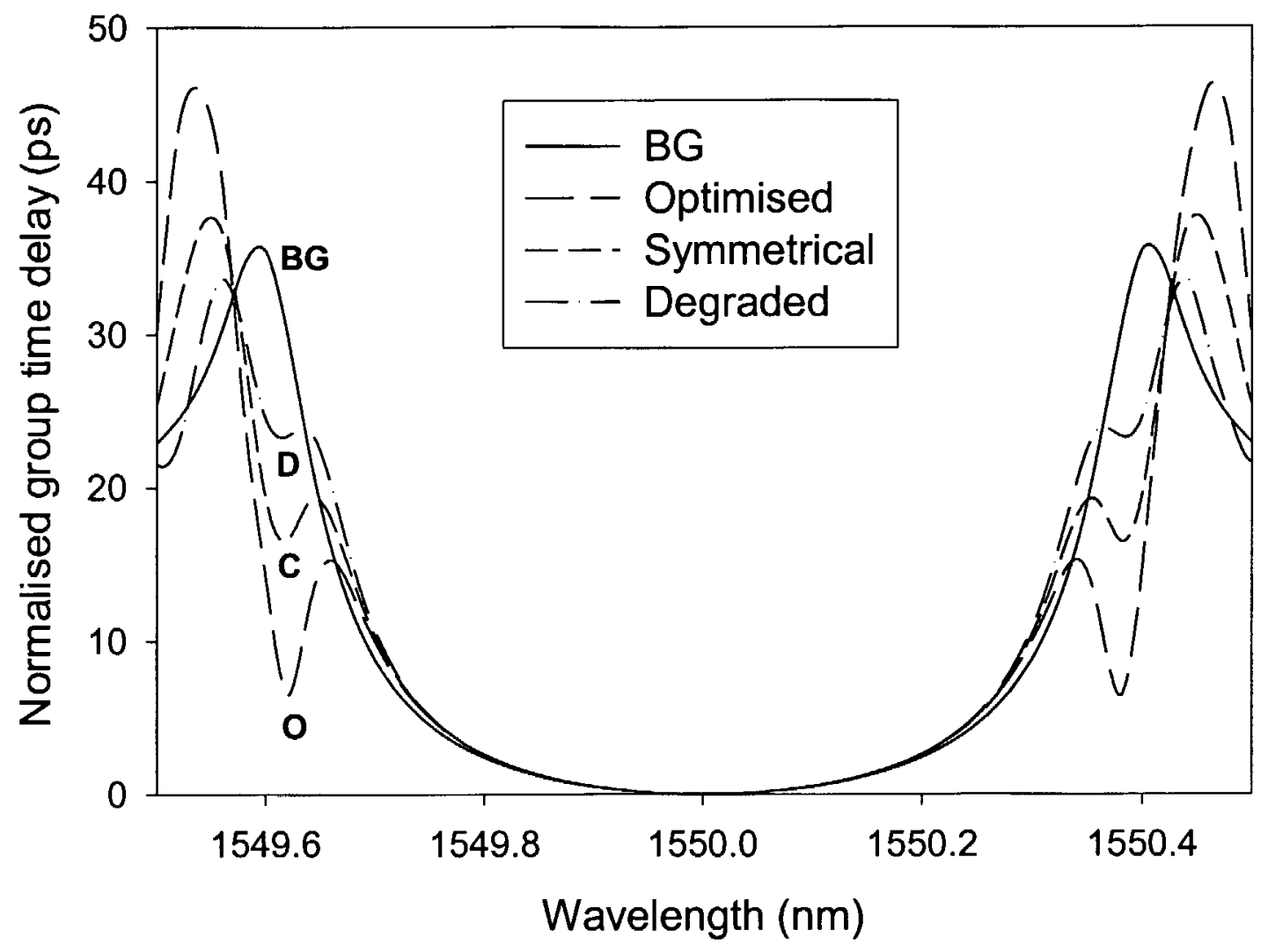

Fig. 6a 


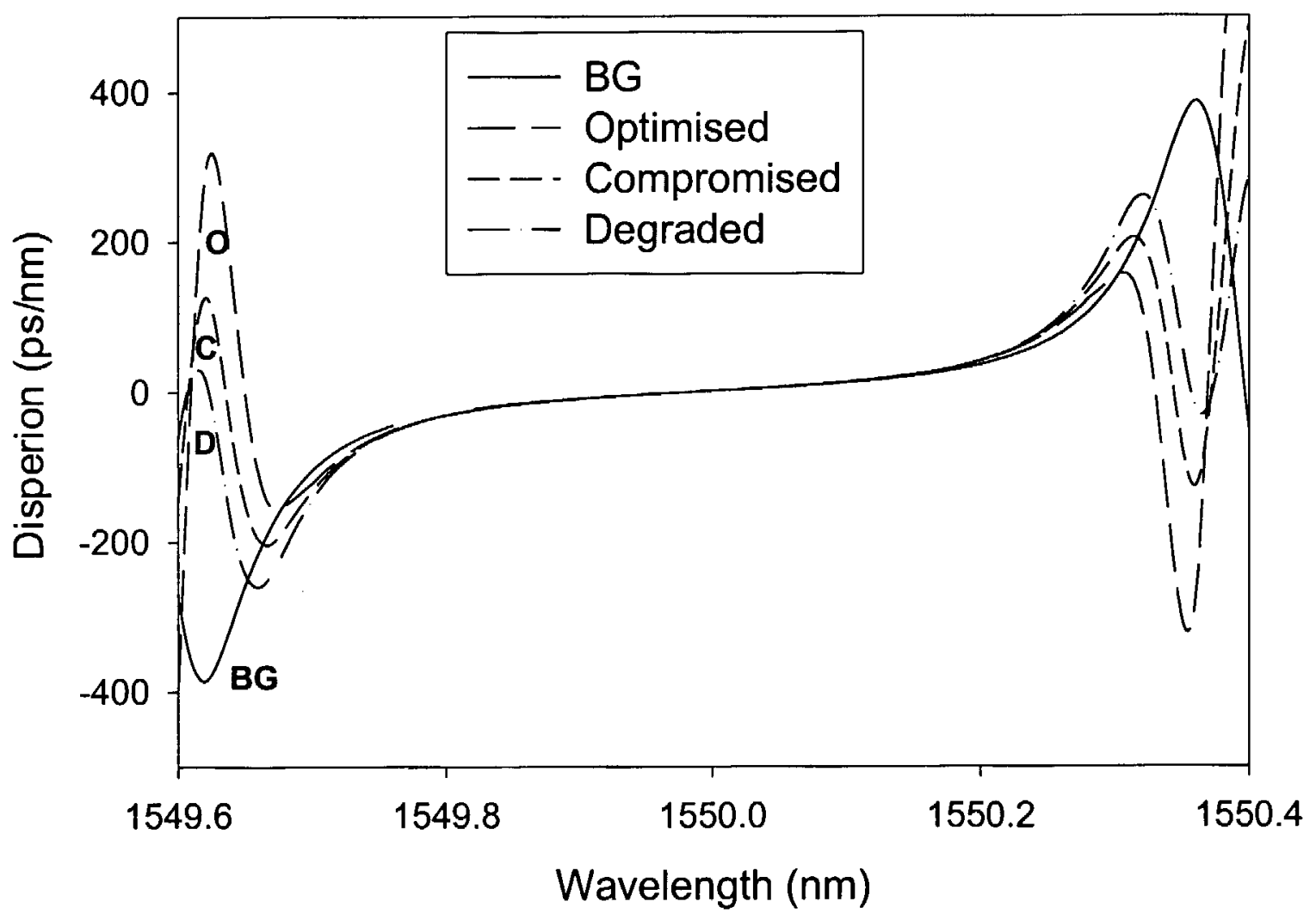

Fig. 6b 
(a)

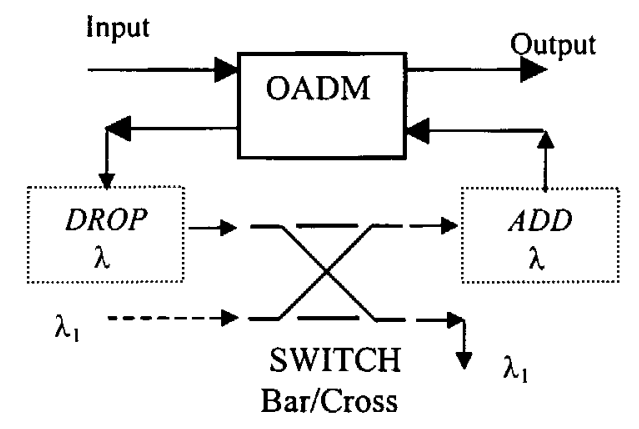

(b)

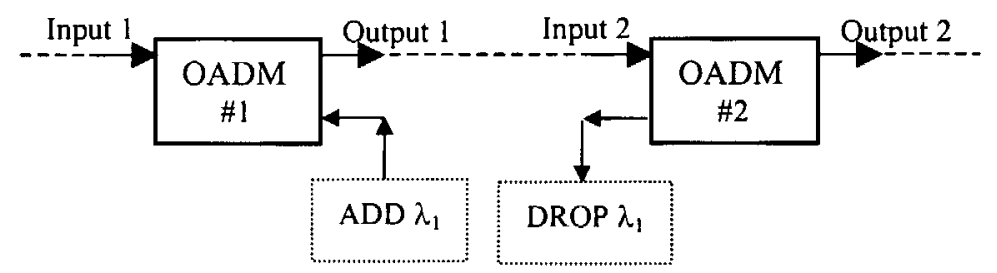

Fig. 7 


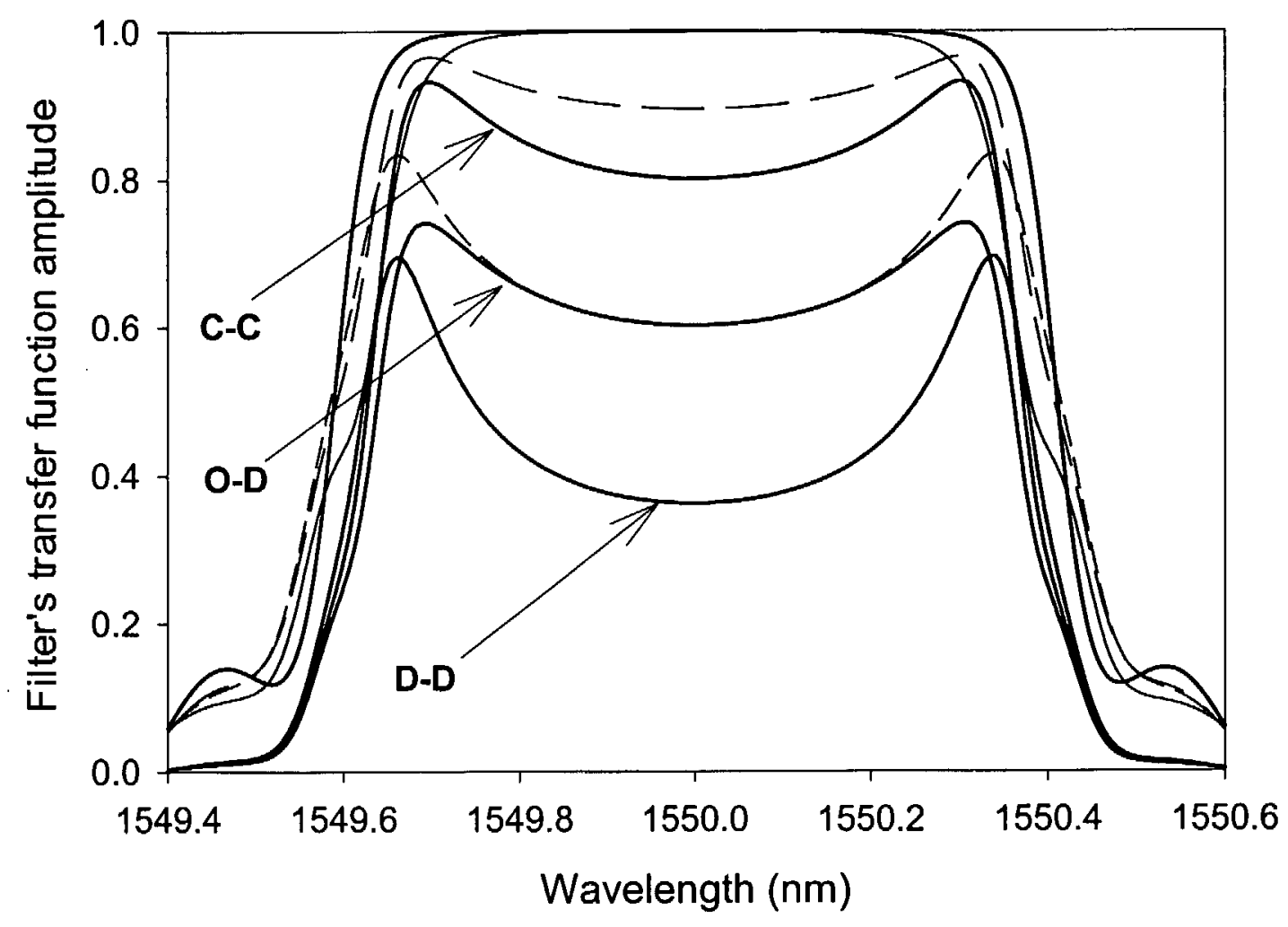

Fig. 8 


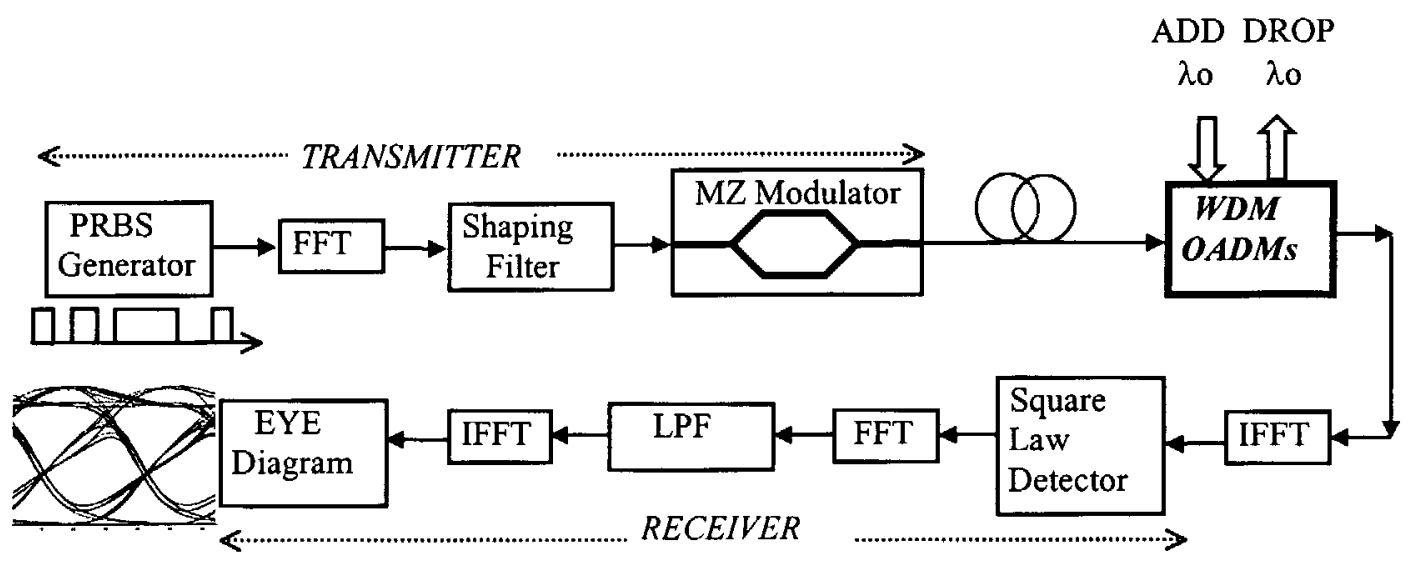

Fig. 9 


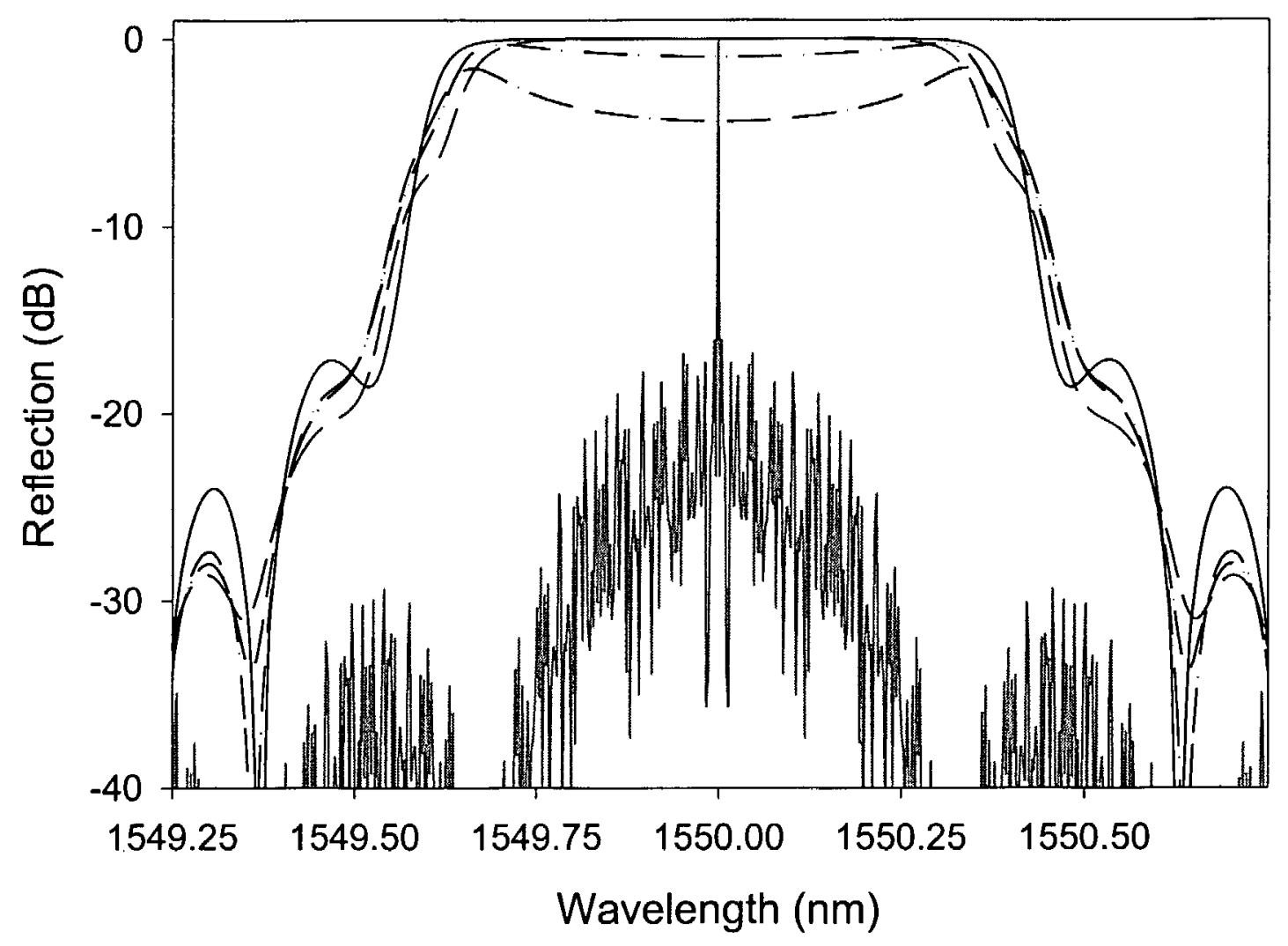

Fig. 10 


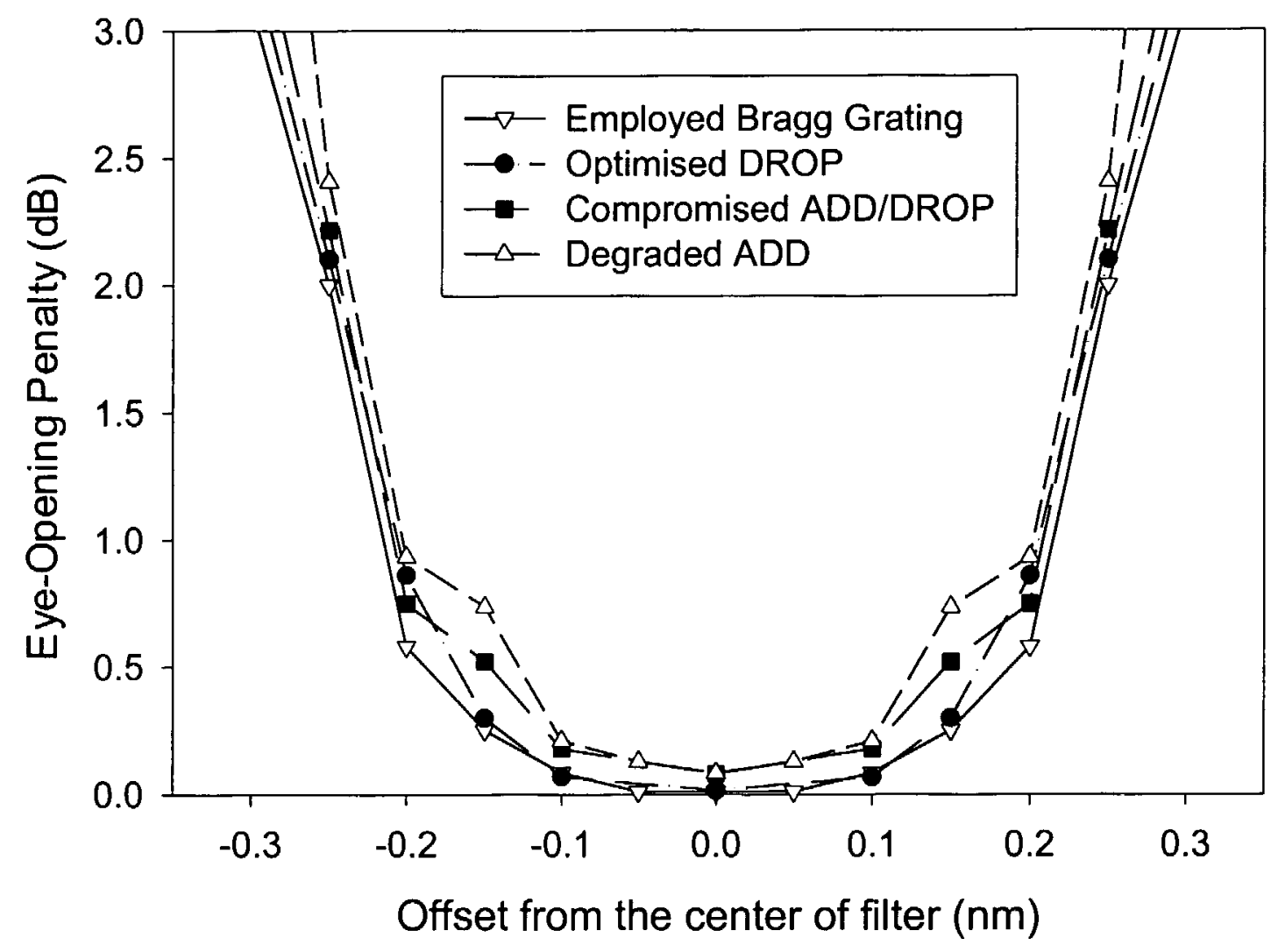

Fig. 11 


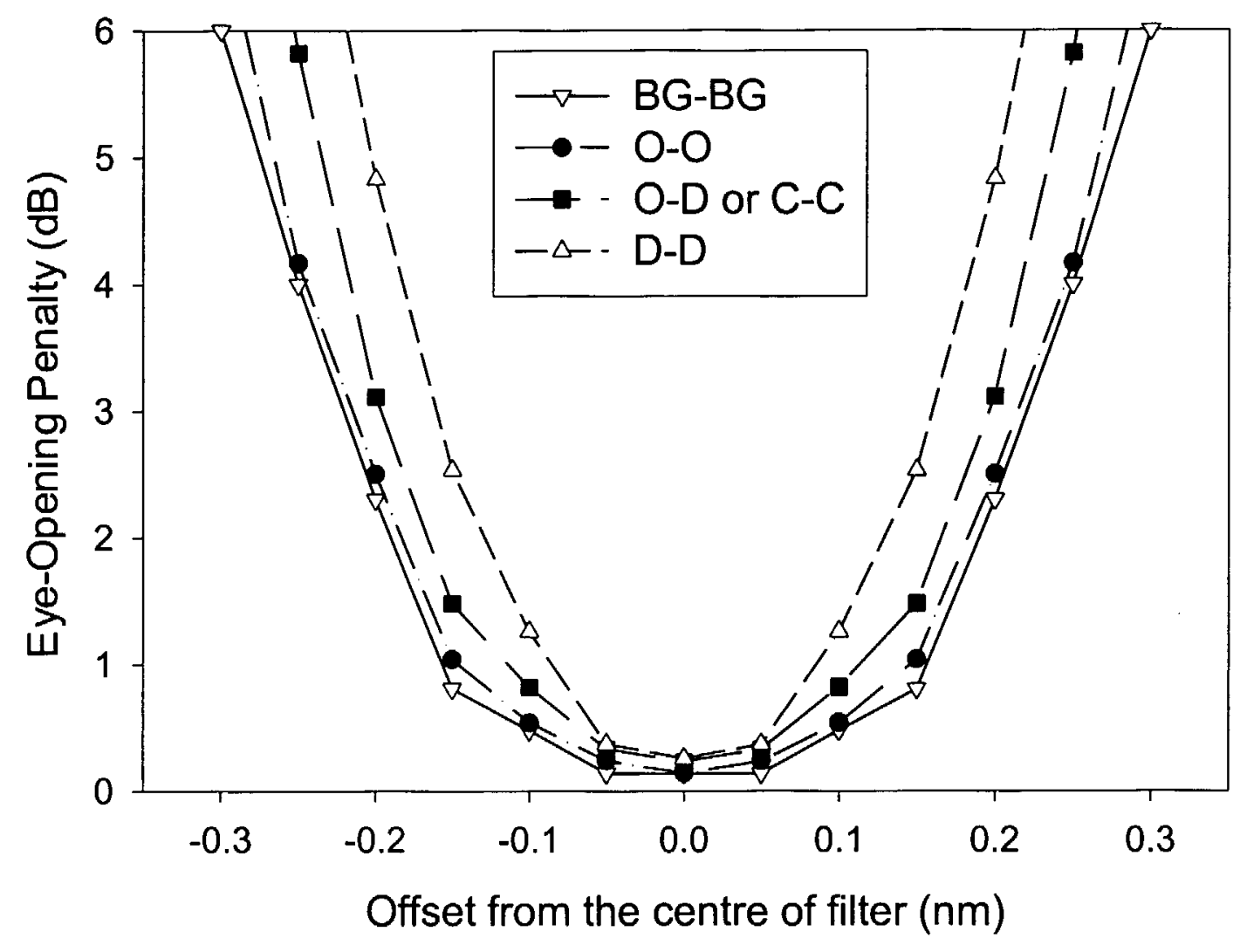

Fig. 12 


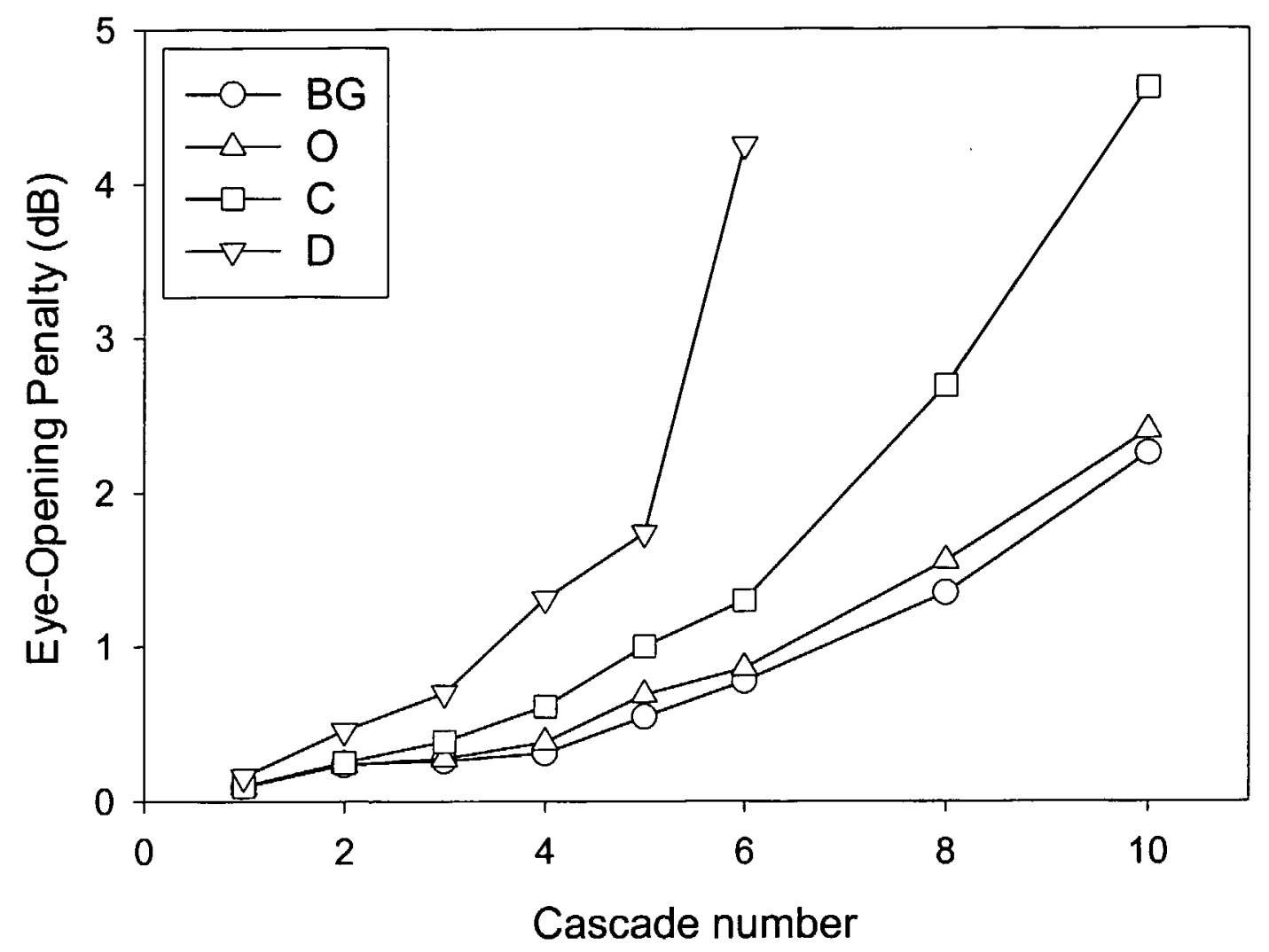

Fig. 13 


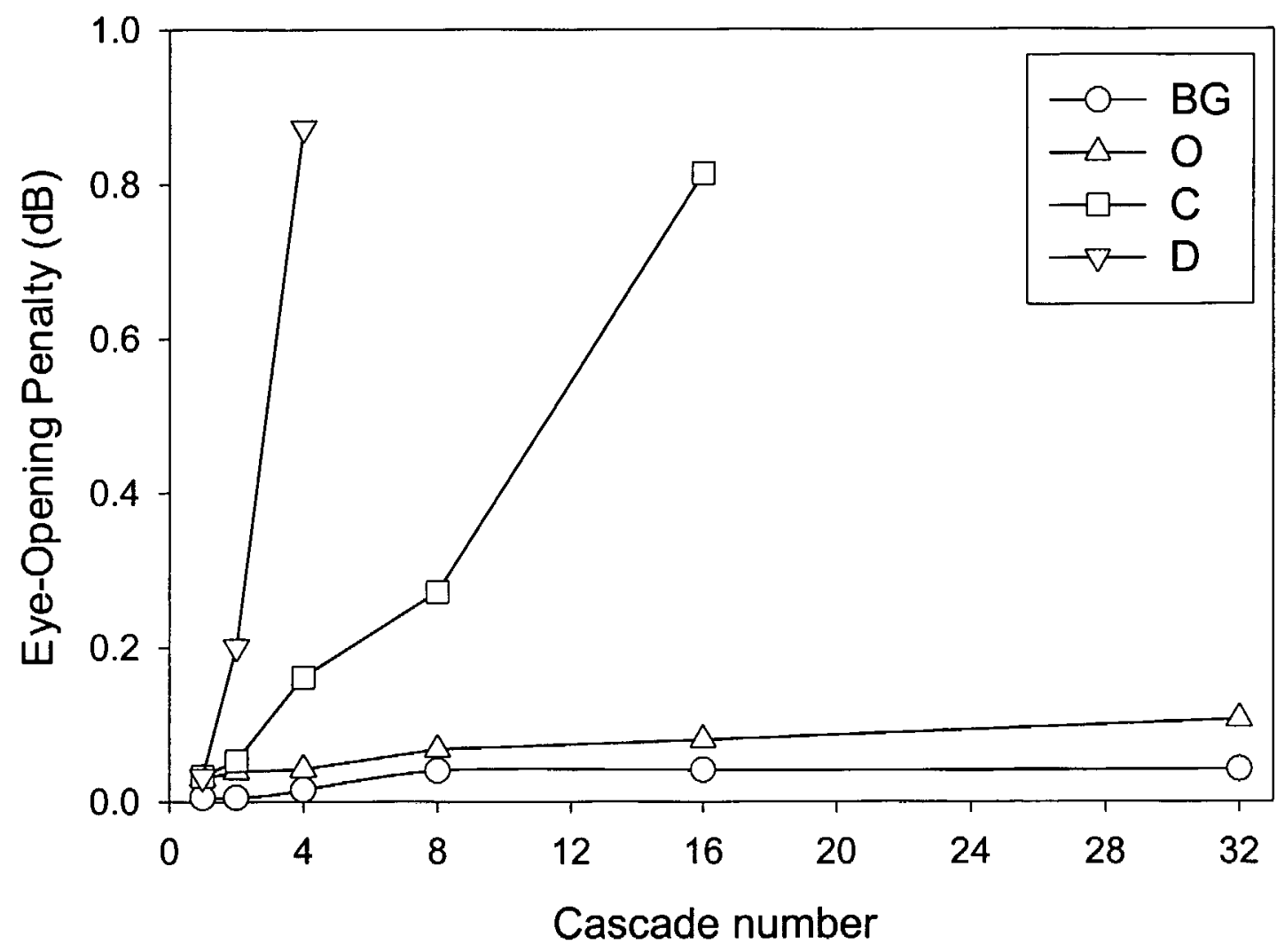

Fig. 14 